\title{
Regional variations in intramyocellular lipid concentration correlate with muscle fiber type distribution in rat tibialis anterior muscle.
}

Citation for published version (APA):

Feyter, H. M., Schaart, G., Hesselink, M. K., Schrauwen, P., Nicolay, K., \& Prompers, J. J. (2006).

Regional variations in intramyocellular lipid concentration correlate with muscle fiber type distribution in rat tibialis anterior muscle. Magnetic Resonance in Medicine, 56(1), 19-25.

https://doi.org/10.1002/mrm.20924

Document status and date:

Published: 01/01/2006

DOI:

10.1002/mrm.20924

Document Version:

Publisher's PDF, also known as Version of record

Document license:

Taverne

Please check the document version of this publication:

- A submitted manuscript is the version of the article upon submission and before peer-review. There can be important differences between the submitted version and the official published version of record.

People interested in the research are advised to contact the author for the final version of the publication, or visit the DOI to the publisher's website.

- The final author version and the galley proof are versions of the publication after peer review.

- The final published version features the final layout of the paper including the volume, issue and page numbers.

Link to publication

\footnotetext{
General rights rights.

- You may freely distribute the URL identifying the publication in the public portal. please follow below link for the End User Agreement:

www.umlib.nl/taverne-license

Take down policy

If you believe that this document breaches copyright please contact us at:

repository@maastrichtuniversity.nl

providing details and we will investigate your claim.
}

Copyright and moral rights for the publications made accessible in the public portal are retained by the authors and/or other copyright owners and it is a condition of accessing publications that users recognise and abide by the legal requirements associated with these

- Users may download and print one copy of any publication from the public portal for the purpose of private study or research.

- You may not further distribute the material or use it for any profit-making activity or commercial gain

If the publication is distributed under the terms of Article 25fa of the Dutch Copyright Act, indicated by the "Taverne" license above, 


\title{
Regional Variations in Intramyocellular Lipid Concentration Correlate With Muscle Fiber Type Distribution in Rat Tibialis Anterior Muscle
}

\author{
Henk M.M.L. De Feyter, ${ }^{1 *}$ Gert Schaart, ${ }^{2}$ Matthijs K. Hesselink, ${ }^{2}$ Patrick Schrauwen, ${ }^{3}$ \\ Klaas Nicolay, ${ }^{1}$ and Jeanine J. Prompers ${ }^{1}$
}

\begin{abstract}
${ }^{1} \mathrm{H}$ MR spectroscopy (MRS) has proved to be a valuable noninvasive tool to measure intramyocellular lipids (IMCL) in research focused on insulin resistance and type II diabetes in both humans and rodents. An important determinant of IMCL is the muscle fiber type, since oxidative type I fibers can contain up to three times more IMCL than glycolytic type II muscle fibers. Because these different muscle fiber types are inhomogeneously distributed in rodent muscle, in the present study we investigated the distribution of IMCL within the rat tibialis anterior muscle (TA) in vivo using single-voxel ${ }^{1} \mathrm{H}$ MRS along with the muscle fiber distribution in the TA ex vivo determined from immunohistological assays. IMCL levels in the TA differed by up to a factor of 3 depending on the position of the voxel. The distribution of IMCL over the TA cross section was not random, but emerged in a pattern similar to the distribution of the predominantly oxidative muscle fiber types. Dietary interventions, such as high-fat feeding and $15 \mathrm{hr}$ of fasting, did not significantly change this typical fiber type-dependent pattern of IMCL content. These results stress the importance of voxel positioning when single-voxel ${ }^{1} \mathrm{H}$ MRS is used to study IMCL in rodent muscle. Magn Reson Med 56:19-25, 2006. (c) 2006 Wiley-Liss, Inc.
\end{abstract}

Key words: intramyocellular lipids; ${ }^{1} \mathrm{H}$ MRS; fiber type; rat; tibialis anterior muscle

Skeletal muscle insulin resistance is one of the earliest detectable aberrations in and a predisposing factor for the development of type II diabetes. Increased content of intramyocellular lipids (IMCL) has been closely associated with decreased whole-body and skeletal muscle insulin sensitivity in both healthy and diabetic humans, as well as in animals (reviewed in Ref. 1). IMCL itself probably does not interfere with the insulin signaling pathway directly; rather, it is considered a surrogate marker of lipid metabolites, such as long chain acyl-CoA, diacylglycerol, and ceramides, that are likely to induce defects in the insulin signaling cascade (2). The good correlation of IMCL with insulin sensitivity led to the suggestion that IMCL could be used as a biomarker for insulin resistance in rat models (3).

${ }^{1}$ Biomedical NMR, Department of Biomedical Engineering, Eindhoven University of Technology, Eindhoven, The Netherlands.

${ }^{2}$ Department of Movement Sciences, Nutrition and Toxicology Research Institute Maastricht, Maastricht University, Maastricht, The Netherlands.

${ }^{3}$ Department of Human Biology, Nutrition and Toxicology Research Institute Maastricht, Maastricht University, Maastricht, The Netherlands.

*Correspondence to: Henk De Feyter, Biomedical NMR, Department of Biomedical Engineering, Eindhoven University of Technology, N-laag b 0.01, P.O. Box 513, 5600 MB Eindhoven, The Netherlands. E-mail: h.m.m.I.d.Feyter@tue.nl

Received 12 January 2006; revised 15 March 2006; accepted 16 March 2006. DOI 10.1002/mrm.20924

Published online 9 June 2006 in Wiley InterScience (www.interscience.wiley. com).

(C) 2006 Wiley-Liss, Inc.
Skeletal muscle stores lipids not only intracellularly, but also extracellularly within interstitial adipocytes. Lipid extraction analysis of muscle biopsy samples therefore cannot differentiate between IMCL and extramyocellular lipids (EMCL) and hence may overestimate the actual IMCL content (1). The discovery that IMCL and EMCL resonate at different frequencies in the ${ }^{1} \mathrm{H} \mathrm{MR}$ spectrum of human skeletal muscle was an important methodological advance in the study of skeletal muscle lipid metabolism (4). The resonance at $1.28 \mathrm{ppm}$ is independent of the angle between the muscle fibers and the static magnetic field, and therefore is attributed to the $\mathrm{CH}_{2}$ groups of IMCL because IMCL is dispersed as spherical droplets within the muscle cells. The other lipid $\mathrm{CH}_{2}$ peak shifts with the angle between the muscle fibers and the field, and is therefore attributed to EMCL, which supposedly is arranged in a sheet-like manner along the muscle fibers. The largest chemical shift difference between the two peaks is $0.2 \mathrm{ppm}$, which is obtained when the muscle fibers are aligned parallel with the magnetic field (5). Since ${ }^{1} \mathrm{H}$ MRS is a noninvasive method, it allows repetitive analysis of IMCL in the same area. Thus, ${ }^{1} \mathrm{H}$ MRS-based IMCL measures are especially useful for determining IMCL levels in human studies, as well as for animal intervention and longitudinal studies.

Using ${ }^{1} \mathrm{H}$ MRS, Neumann-Haefelin et al. (6) showed that the IMCL content in rat depends on age, strain, and gender, which complicates comparisons among different rat groups and different studies. They also showed that the rather oxidative soleus muscle (SOL) has a higher IMCL content than the predominantly glycolytic tibialis anterior (TA) $(6,7)$. Similar differences have been observed in human calf skeletal muscles, and the SOL has been reported to contain about three times more IMCL than the TA $(8,9)$. This can be attributed to differences in the muscle fiber type composition of the different muscles, since it is well documented that the oxidative type I muscle fibers can contain up to three times more IMCL than the glycolytic type II muscle fibers $(10,11)$.

Variations in fiber type composition can explain differences in IMCL levels found among different muscles studied as a whole. However, rodent muscle can also show a marked regionalization of the different muscle fiber types within a specific muscle itself, in both the cross-sectional plane and the longitudinal direction (12). Wang and Kernell (12) showed a pronounced regionalization of type I muscle fibers in cross sections of the TA of the rat. These type I fibers were clustered in the medial-posterior part of the muscle, close to the tibia bone. The pronounced regionalization of type I muscle fibers in the rat TA and the 
higher capacity of type I fibers to store IMCL compared to type II fibers suggest that IMCL levels may vary significantly within the cross section of the rat TA. This may imply that differences in voxel positioning within the rat TA can cause a large variability in the IMCL signal measured by single-voxel ${ }^{1} \mathrm{H}$ MRS, which could result in a misjudgment of changes in IMCL content during longitudinal and/or interventional studies in rats. Therefore, the aim of the present study was to examine the IMCL content at different positions in the TA of Wistar rats and relate it to muscle fiber type composition as assessed by immunohistological analysis.

Furthermore, we determined the IMCL content at different positions within the TA after the rats were subjected to starvation and high-fat feeding. Neumann-Haefelin et al. (7) showed that the increase in plasma free fatty acids (FFA) during starvation was paralleled by muscle-typedependent changes in IMCL content in the rat, when muscles were studied as a whole. To examine whether interventions with predicted increases in blood FFA (fasting and a high-fat diet) affected IMCL within the TA in a fiber-type-specific manner, we examined IMCL content after $15 \mathrm{hr}$ of fasting and after consumption of a high-fat diet, and assessed its relation to muscle fiber typology.

\section{MATERIALS AND METHODS}

\section{Animals and Study Design}

Experiments were performed on 14 male Wistar rats $(\sim 16$ weeks old, mean body weight \pm standard error of the mean $(\mathrm{SEM})=0.375 \pm 0.007 \mathrm{~kg})$. The animals were housed in pairs at $20^{\circ} \mathrm{C}$ and $50 \%$ humidity, on a 12-hr light-dark cycle, with ad libitum access to normal chow and water during the time preceding the experiments. All experimental procedures were approved by the Animal Ethics Committee of Maastricht University.

Twelve animals were subjected to two single-voxel ${ }^{1} \mathrm{H}$ MRS measurements separated by 7 days. All animals were in the fed state unless stated otherwise. Six animals were put on a high-fat diet $(\sim 16 \%$ fat, $\sim 50 \%$ carbohydrate, $\sim 18 \%$ protein, diet W402106; Arie Blok Dieetvoeding, Woerden, The Netherlands) ad libitum for 7 days after the first measurement. The remaining six animals stayed on normal chow but were fasted for $15 \mathrm{hr}$ preceding the second measurement. Both MRS sessions were performed at identical time points starting at 8 a.m. and lasted approximately $4.5 \mathrm{hr}$. The animals were randomly assigned to the dietary intervention groups.

During the MR experiments the animals were anesthetized using isoflurane (Forene®, 1-2\%) in a mixture of $\mathrm{O}_{2}$ and $\mathrm{N}_{2} \mathrm{O}(0.15: 0.30 \mathrm{~L} / \mathrm{min})$ and body temperature was maintained at $37^{\circ} \mathrm{C} \pm 1^{\circ} \mathrm{C}$ using a heating pad. Respiration was monitored using a pressure sensor that registered the movement of the thorax (ECG trigger unit; Rapid Biomedical, Wurzburg, Germany), and blood oxygenation and heart rate were measured with a pulse oximeter (Nonin 8600V; Nonin Medical, Inc., Plymouth, MN, USA).

At the end of the second MRS measurement, the anesthetized animals were killed by cervical dislocation. The TA from the hindleg measured during the MRS experiment was rapidly excised, and the midbelly region was

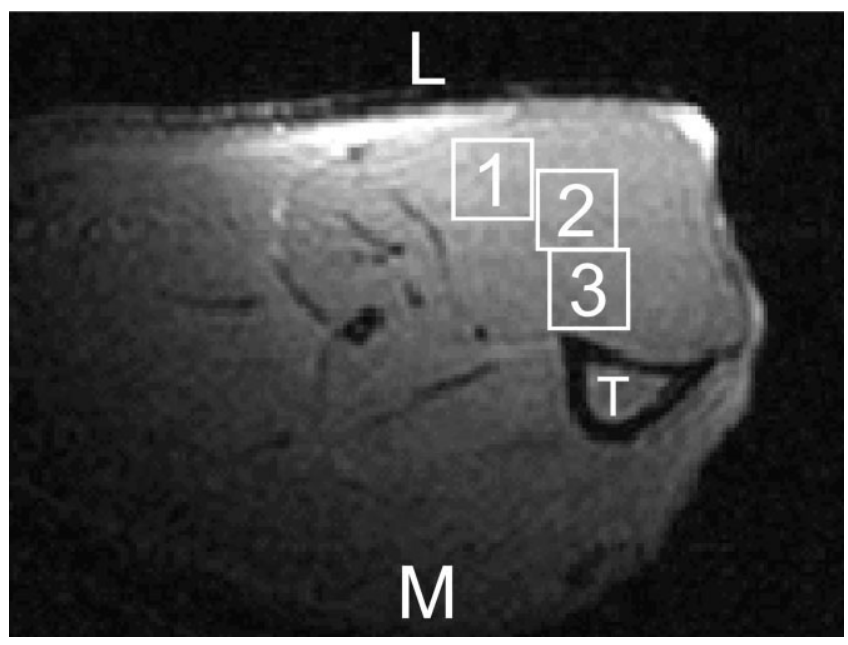

FIG. 1. Transversal adiabatic spin-echo image of the midbelly region of a rat lower hindleg with voxel positioning within the TA muscle for single-voxel ${ }^{1} \mathrm{H}$ MRS. $M=$ medial, $\mathrm{L}=$ lateral, $\mathrm{T}=$ tibia bone. Acquisition parameters: TR $=2 \mathrm{~s}$, TE $=24 \mathrm{~ms}$.

frozen in melting isopentane and stored at $-80^{\circ} \mathrm{C}$ until it was used for immunohistological analysis.

Two animals were used to study the reproducibility of the ${ }^{1} \mathrm{H}$ MRS measurement (see below).

\section{MR Experiments}

\section{Single-Voxel ${ }^{1} \mathrm{H}$ MRS}

All MR experiments were performed on a 6.3 Tesla horizontal bore Varian MR system using an ellipsoid ${ }^{1} \mathrm{H}$ surface coil (18/22 mm). In all rats we measured the SOL and TA, always starting with the SOL and repositioning the animal between measurements. The animal was positioned laterally on the heating pad with the lateral side of the lower hindleg on the ${ }^{1} \mathrm{H}$ surface coil. The leg was positioned in such a way that the largest cross section of the SOL or TA, respectively, was in the isocenter of the magnet and the muscle fibers were aligned with the static magnetic field. Transversal images of the midbelly region of the muscles were acquired using an adiabatic spin-echo sequence (pulse repetition time $(\mathrm{TR})=2 \mathrm{~s}$, echo time $(\mathrm{TE})=24 \mathrm{~ms}$ ) to achieve proper placement of the spectroscopic regions of interest (ROIs).

Single-voxel localized ${ }^{1} \mathrm{H}$ MR spectra were acquired using the localization by adiabatic selective refocusing (LASER) sequence (13) with additional outer volume suppression $(\mathrm{TR}=1 \mathrm{~s}$, TE $=28 \mathrm{~ms}$, spectral width $=4000 \mathrm{~Hz}$, number of data points $=2048$, sequence for water suppression with adiabatic modulated pulses (SWAMP) water suppression (14), 2048 averages for SOL, 1024 averages for TA). The voxel size was $1.3 \times 1.3 \times 2 \mathrm{~mm}^{3}$ for the SOL, and $2 \times 2 \times 2 \mathrm{~mm}^{3}$ for the TA. In the SOL one voxel was measured, whereas for the TA voxels were placed at three different positions within the muscle cross section: (TA1) lateral, (TA2) in the center, and (TA3) medial, close to the tibia bone (Fig. 1). Unsuppressed water spectra (128 averages for SOL, 64 averages for TA) were recorded from the same voxels and used as internal reference. Furthermore, a series of eight unsuppressed water spectra were recorded 
with varying TEs (32 averages) to determine the $T_{2}$ of muscle water. The reproducibility of the IMCL quantification in the TA cross-section was determined in one rat, measured on 3 different days within 1 week. In one other animal, the IMCL content at the described voxel positions was studied in four different cross-sectional planes of the TA, going from the knee toward the ankle, to analyze IMCL variations in the longitudinal direction of the muscle.

\section{Spectroscopic Imaging (SI)}

SI was performed on one rat only. Because in control rats the IMCL content in the TA is low, the SI data were collected from a rat that had been on the high-fat diet for 7 days. A 2D SI sequence with LASER volume preselection was used. The slice ( $3 \mathrm{~mm}$ thick) was selected in the transverse orientation. The position and size of the LASER box were adjusted to exclude subcutaneous fat and bone marrow as much as possible, while covering most of the TA muscle. The spectra were acquired with $\mathrm{TR}=1 \mathrm{~s}$, $\mathrm{TE}=$ $29 \mathrm{~ms}$, SWAMP water suppression, and 48 averages. A matrix of $8 \times 8$ was used over a field of view (FOV) of $5 \times$ $5 \mathrm{~mm}^{2}$, resulting in a scan time of $51 \mathrm{~min}$. Unsuppressed water spectra were recorded with identical settings, except that four scans were averaged.

\section{Data Analysis}

For the single-voxel spectra no further postprocessing was applied, except for manual phasing. For the SI data a Hanning filter was applied in both spatial dimensions and spatial zero-filling was applied to $16 \times 16$ points, resulting in a nominal voxel size of $0.29 \mu \mathrm{l}$. All spectra were fitted in the time domain by using a nonlinear least-squares algorithm (AMARES) in the jMRUI software package (15). The unsuppressed water spectrum was phased and fitted to a Lorentzian line shape. The zero-order phase correction from the water spectrum was applied to the corresponding water-suppressed ${ }^{1} \mathrm{H}$ spectrum, and the total creatine ( $\mathrm{tCr}$ ) $\mathrm{CH}_{3}$ peak was referenced to $3.02 \mathrm{ppm}$. In the water-suppressed spectrum, the central line of the $\mathrm{tCr} \mathrm{CH}_{3}$ peak and the IMCL $\mathrm{CH}_{2}$ peak (1.28 ppm) was fitted to Gaussian line shapes. The tCr linewidth $\left(L W_{t C r}\right)$ and IMCL linewidth $\left(L W_{I M C L}\right)$ were constrained with respect to the linewidth of the water peak $\left(L W_{\mathrm{H}_{2} \mathrm{O}}\right)$ according to

$$
L W_{t C r}=-0.71+0.49 \cdot L W_{\mathrm{H}_{2} \mathrm{O}}
$$

and

$$
L W_{I M C L}=4.06+1.04 \cdot L W_{\mathrm{H}_{2} \mathrm{O}}
$$

The constraint for the tCr linewidth was derived from previously measured and present data sets featuring a well-resolved triplet for the $\mathrm{tCr} \mathrm{CH}_{3}$ resonance at $3.02 \mathrm{ppm}$ (number of data sets $=74, \mathrm{R}=0.735, P<0.0001$, not shown). The constraint for the IMCL linewidth was derived from data sets that were uncontaminated with EMCL (number of data sets = 91, $\mathrm{R}=0.537, P<0.0001$, not shown).

The IMCL and tCr levels were expressed as a percentage of the water signal measured in the same voxel. For the SI data set, the water and IMCL signals in the voxels from the two leftmost columns and the two uppermost rows do not originate from the same location, due to the water-fat shift. Therefore, these voxels were discarded, resulting in $14 \times$ 14 usable voxels.

\section{Immunohistology}

The TA muscles of five animals from in the dietary intervention study were used for immunohistological staining. Cryostat sections (5 $\mu \mathrm{m}$ thick) of midbelly muscle were thaw-mounted on uncoated glass slides. Air-dried cryosections were treated with $0.5 \%$ Triton X-100 (Merck) in phosphate-buffered saline (PBS) for $5 \mathrm{~min}$, washed with three exchanges of PBS for $5 \mathrm{~min}$, and processed for tripleimmunofluorescence staining. In brief, sections were incubated for $45 \mathrm{~min}$ at room temperature with a mixture of primary antibodies: a rabbit polyclonal antiserum against the basement membrane protein laminin (L-9393; Sigma, Zwijndrecht, The Netherlands), a monoclonal antibody against myosin heavy chain 1 (A4.840), and a monoclonal $\mathrm{IgG}_{1}$ antibody against myosin heavy chain 2A (N2.261). The latter two antibodies were developed by Dr. Helen Blau and obtained from the Developmental Studies Hybridoma Bank under the auspices of the NICHD and maintained by the Department of Biological Sciences, University of Iowa, Iowa City, IA, USA. Antibodies were diluted in $0.05 \%$ Tween 20 (Sigma) in PBS. Thereafter the sections were washed three times with PBS for $5 \mathrm{~min}$ and incubated for $30 \mathrm{~min}$ at room temperature with the appropriate secondary antibody mixture diluted in $0.05 \%$ Tween $20 /$ PBS. The sections were then washed again three times for 5 min with PBS and mounted in Mowiol.

Sections were examined using a Nikon E800 fluorescence microscope coupled to a Basler A101C progressive scan color charge-coupled device camera. Images were captured for every single color (red, green, and blue) from all sections, processed, and analyzed using Lucia GF 4.80 software (Lucia, Czech Republic). Special care was taken to use the same camera settings (gain and exposure time) while grabbing images.

\section{Statistics}

The results are presented as mean \pm SEM. The normality of the data was examined by means of the Shapiro-Wilk test. One-way analysis of variance (ANOVA) with a Bonferroni post-hoc test was used to compare the IMCL and tCr values from the different voxel positions. The effect of the different dietary interventions was evaluated using a paired Student's $t$-test. $P<0.05$ was considered statistically significant.

\section{RESULTS}

\section{Single-Voxel ${ }^{1} \mathrm{H}$ MRS}

The typical linewidths of the water peak after localized shimming were $14 \mathrm{~Hz}$ or $0.052 \mathrm{ppm}$ at 6.3 Tesla. The SWAMP water suppression performed very well, so it was not necessary to use postprocessing techniques to remove residual water signal. Figure 2 shows typical single-voxel water-suppressed ${ }^{1} \mathrm{H}$ spectra from the TA. The orientationdependent dipolar splitting of the $\mathrm{tCr} \mathrm{CH}_{2}$ resonance at 


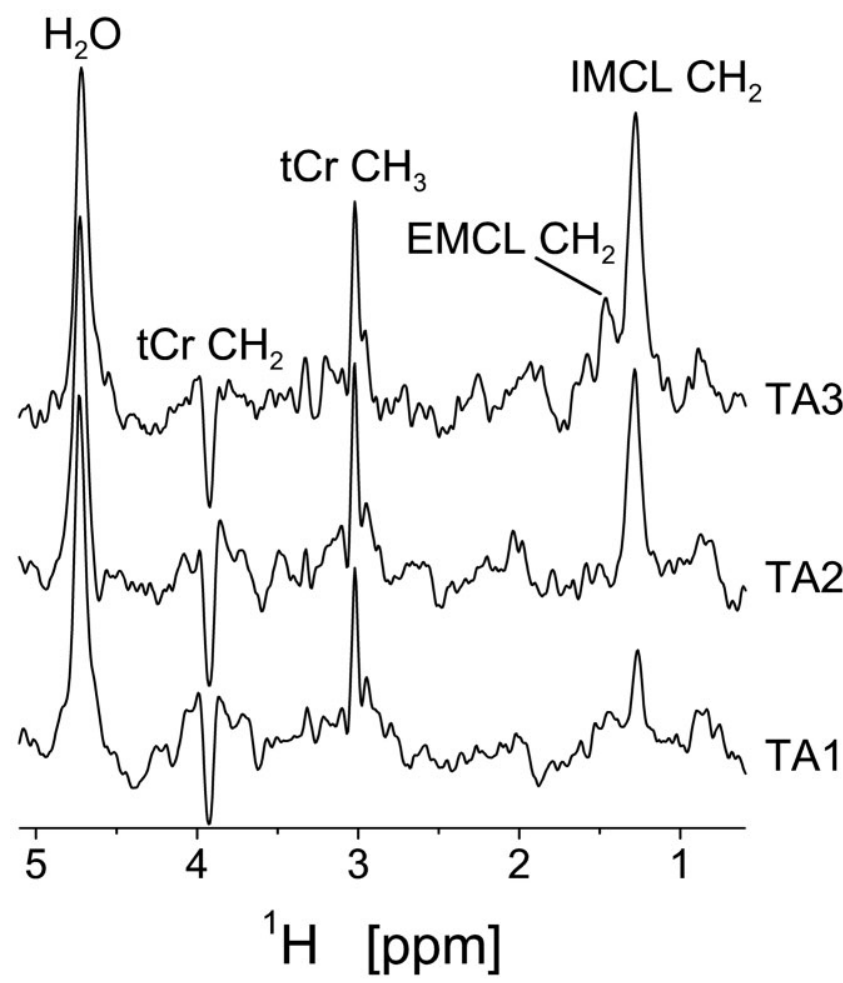

FIG. 2. Typical examples of single-voxel localized ${ }^{1} \mathrm{H}$ MR spectra using LASER and SWAMP water suppression from voxel positions 1-3 in rat TA muscle. Acquisition parameters: $T R=1 \mathrm{~s}, \mathrm{TE}=28 \mathrm{~ms}$, 1024 averages, voxel size $=2 \times 2 \times 2 \mathrm{~mm}^{3}$.

$3.92 \mathrm{ppm}$ (doublet) and $\mathrm{tCr} \mathrm{CH}_{3}$ resonance at $3.02 \mathrm{ppm}$ (triplet) confirm the proper positioning of the rat hindleg. The majority of the spectra did not contain an observable EMCL peak. For the spectra that did contain an EMCL signal, the IMCL and EMCL peaks were well separated $(\sim 0.15 \mathrm{ppm})$.

Figure 3 shows the IMCL content for the different voxel positions in the TA and the SOL measured in 12 rats during the first MRS session. IMCL was remarkably heterogeneously distributed within the cross section of the TA. The IMCL levels displayed a typical pattern related to the voxel position from which the signal originated: IMCL values were lowest in the lateral region (voxel position 1) and highest in voxel position 3 , close to the tibia bone, while the IMCL level at voxel position 2 , in the center of the TA cross-section, was between the values of voxel positions 1 and 3. The IMCL contents at the different TA voxel positions all differed significantly from each other $(N=12$; TA 1 vs. TA2, $P=0.022$; TA1 vs. TA3, $P<0.001$; TA2 vs. TA3, $P=0.007)$. The IMCL content of the SOL was significantly different from the IMCL content of the TA at voxel positions $1(N=12 ; P=0.003)$ and $3(N=12 ; P=$ 0.047 ), but was similar to the IMCL content of the TA at voxel position 2 .

The tCr content of the TA (also shown in Fig. 3) did not change as a function of voxel position, and for each voxel position it showed a smaller variation (SEM) compared to IMCL $(N=12)$. The tCr content at any voxel position in the TA was higher than that in the $\operatorname{SOL}(N=12$, all $P \leq 0.001)$.
The reproducibility of the IMCL determination at the different voxel positions within the TA was measured in one rat, which was not included in the dietary-intervention study. The three typical voxel positions used in the TA were analyzed on 3 different days within 1 week. The coefficient of variance of the IMCL measurement was $16.7 \%$ for TA $1,16.7 \%$ for TA 2 , and $14.5 \%$ for TA3. The reproducibility of the $\mathrm{tCr}$ quantification varied from $7.6 \%$ for TA1 to $10.3 \%$ for TA2 and $14.3 \%$ for TA3. The analysis of variations in IMCL content in the longitudinal direction of the TA, measured in a second rat that was not included in the dietary-intervention study, revealed that the pattern shown in Fig. 3 was constant in the midbelly region (middle part) of the muscle (data not shown).

\section{SI}

SI was performed to visualize the spatial IMCL distribution within the TA cross section at a higher resolution. Figure 4 shows the IMCL levels for the $14 \times 14$ voxels from the SI data set $(N=1)$. The color-coded IMCL image was overlaid on a coregistered MR image for anatomical guidance. The SI region covered most of the TA cross section and confirmed the inhomogeneous distribution of IMCL. A clear gradient was observed in the IMCL levels, with the highest values in the medial-posterior part, close to the tibia bone, and the lowest values in the peripheral anterior region.

\section{Immunohistology}

To clarify the origin of the spatial differences in IMCL concentration, we performed immunohistological analyses on muscle cross-section regions corresponding to the voxel positions used for the single-voxel ${ }^{1} \mathrm{H}$ MRS. Examples of the immunohistological images are depicted in Fig. 5. The immunohistological staining shows basal lamina in blue, type I muscle fibers in red, and type IIa fibers in green. Fibers without any staining represent the IIb muscle fiber type. The tissue displayed in the left panel of Fig. 5 (TA1) contained only a few type IIa fibers and no type I

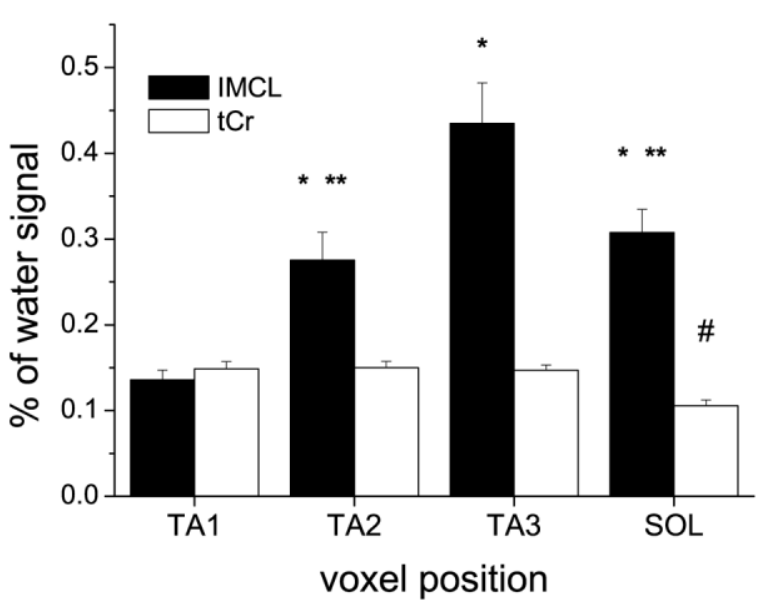

FIG. 3. IMCL and $\mathrm{tCr}$ content relative to the water signal as a function of voxel position. Data are presented as mean $(N=12) \pm$ SEM. * $P<0.05$ relative to voxel position TA1, ${ }^{* *} P<0.05$ relative to voxel position TA3, \# $P<0.05$ relative to voxel position TA1, TA2, and TA3. 


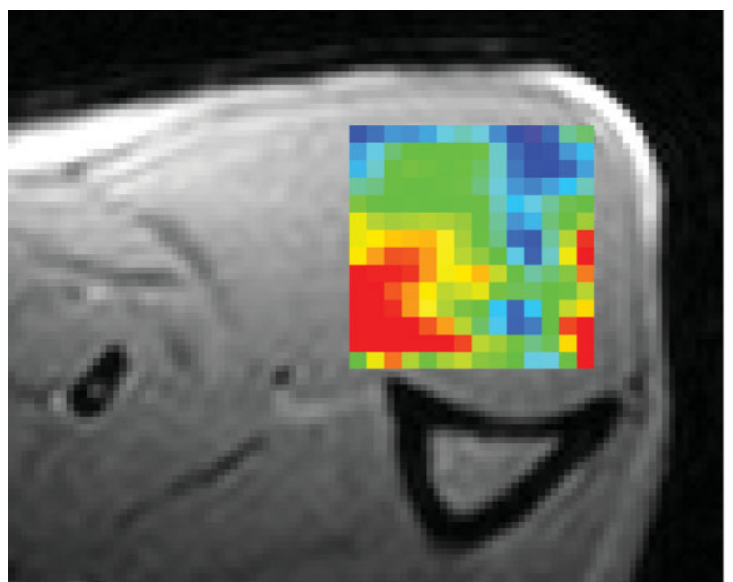

FIG. 4. IMCL levels in the $14 \times 14$ voxels of the SI data set on top of a coregistered MR image. Color coding represents the IMCL as a percentage of the water signal. Acquisition parameters: TR $=1 \mathrm{~s}$, $\mathrm{TE}=29 \mathrm{~ms}, 48$ averages, voxel size $=0.31 \times 0.31 \times 3 \mathrm{~mm}^{3}$.

fiber. This part of the TA muscle was composed mainly of the nonoxidative type IIb fibers. The tissue in the middle panel (TA2) contained a few type I fibers and some more type IIa fibers, but was still dominated by nonoxidative fibers. The tissue shown in the right panel (TA3) was dominated by the oxidative type I and type Ila fibers. In Fig. $6 a-c$ the average IMCL content from different voxel positions is displayed as a function of the average percentage of type I, IIa, and IIb muscle fibers within these similar voxel positions, respectively. The muscle fiber type quantification was performed in the histological regions corresponding to the three MRS voxel positions. The IMCL data and the immunohistological data are from the same animals $(N=5)$. A larger amount of oxidative type I and type IIa fiber was paralleled by a higher IMCL level, and the relative amount of glycolytic IIb fibers showed a negative relationship with IMCL.

\section{Dietary Interventions}

IMCL can change during and after interventions that induce increased plasma FFA, such as a high-fat diet and fasting. We examined the influence of both dietary interventions on the IMCL content in TA and SOL and the IMCL distribution within the TA. The high-fat diet resulted in a substantial increase in IMCL in all voxels of the TA $(N=6$; post-diet vs. pre-diet; TA1: $+115 \%, P=0.008$;
TA2: $+120 \%, P=0.001$; TA3: $+111 \%, P=0.002)$ as well as in the SOL (post-diet vs. pre-diet; SOL: $+67 \%, P=$ 0.036) (Fig. 7a). In contrast, the 15 -hr fasting procedure did not induce any significant changes in IMCL for the voxels in the TA $(N=5$; one dropout due to failing heating pad; Fig. 7b). The fasting procedure did result in a decrease of IMCL in the SOL (post-diet vs. pre-diet; SOL: $-40 \%, P=$ 0.004). Neither dietary intervention affected the tCr content in the TA or SOL (data not shown).

The dietary interventions had no effect on the $T_{2}$ of muscle water measured in the different voxels (data not shown), which justifies the use of the water signal to quantify the IMCL and tCr content.

\section{DISCUSSION}

Using noninvasive MR techniques combined with invasive examination of the spatial heterogeneity of rat muscle fiber typology, we conclusively showed that spatial differences in IMCL content are accounted for by muscle fiber type distribution. Strikingly, the increase in IMCL content upon consumption of a high-fat diet was similar across all fiber types, preserving the pronounced pattern of IMCL distribution. This study therefore underscores the importance of anatomical positioning of the voxel of interest in rat muscle, in both cross-sectional and longitudinal studies.

${ }^{1} \mathrm{H}$ MRS is a well-established noninvasive method to measure IMCL, and is frequently used to study lipid storage in human skeletal muscle in relation to exercise, insulin resistance, and type II diabetes. ${ }^{1} \mathrm{H}$ MRS has also become an important tool for research on insulin resistance and type II diabetes in rat studies. In rat studies, ${ }^{1} \mathrm{H}$ MRS is often applied to the TA, among other aspects, because of its characteristic muscle fiber architecture that maximizes the separation of IMCL and EMCL peaks. The typical volume used in single-voxel ${ }^{1} \mathrm{H}$ MRS in rat TA is about $8 \mu$, which is small relative to the total volume of the TA $( \pm 500 \mu \mathrm{l})$ and can therefore be representative of the whole muscle only if the muscle itself displays a homogeneous IMCL distribution. Since it is known that muscle fiber type is an important determinant of IMCL content and that muscle fiber types can be strongly regionalized in rat muscle, we examined the spatial distribution of IMCL and correlated this with immunohistological analyses of muscle fiber typology within the rat TA cross section.

When fat storage in rat skeletal muscle is studied, the IMCL is usually normalized to the tCr signal $(3,6,7,16,17)$. In human studies, however, the IMCL is often expressed as
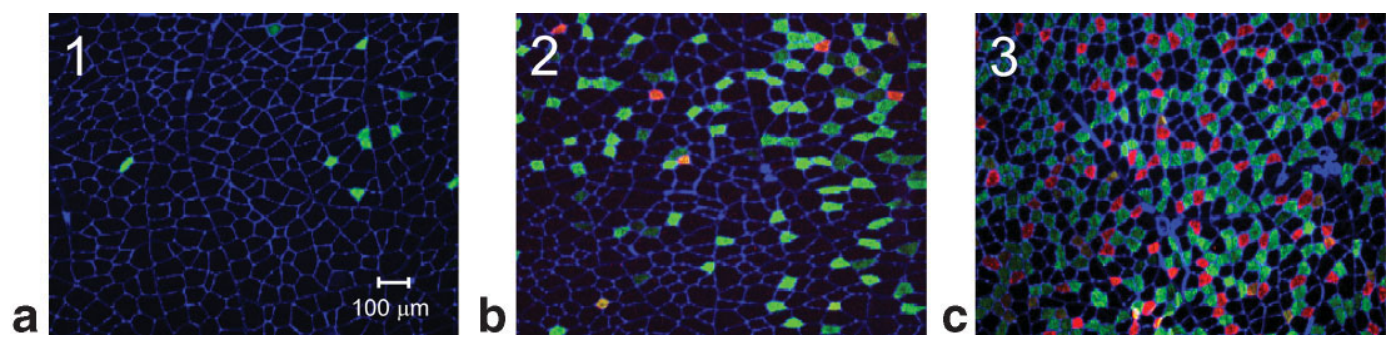

FIG. 5. Triple-immunofluorescence assay in representative areas $\left(1.36 \times 1.07 \mathrm{~mm}^{2}\right)$ of the TA corresponding to voxel positions $1-3$. Blue: basal lamina; red: type I muscle fibers (slow oxidative); green: type lla muscle fibers (fast oxidative). 

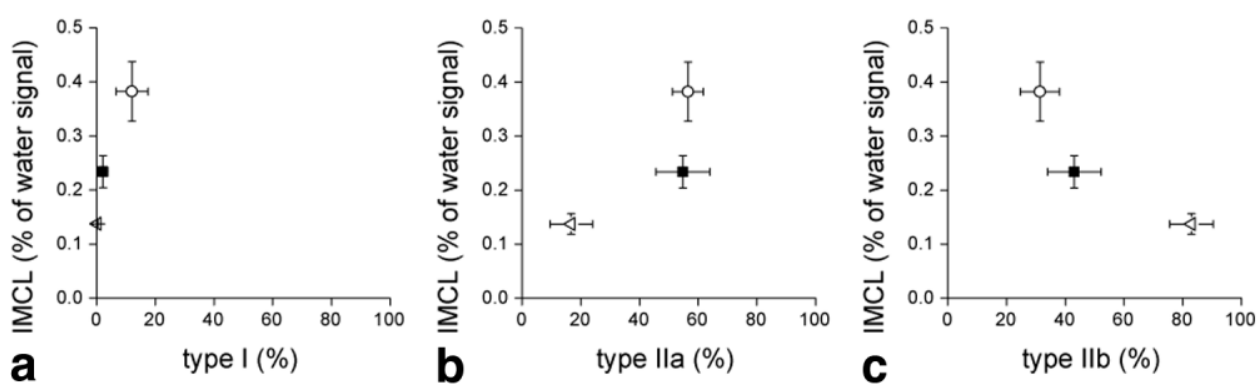

FIG. 6. IMCL content in the TA plotted as a function of the muscle fiber type. Each data point represents the average IMCL content from the different voxel positions ( $\triangleleft=\mathrm{TA} 1 ; \mathbf{\square}=\mathrm{TA} 2 ; \bigcirc=\mathrm{TA} 3$ ) and the corresponding percentage of type I (a), type lla (b), and type Illb (c) fibers $(N=5)$. Error bars represent SEM.

a percentage of the water signal originating from the same voxel, since the tCr signal is small compared to the IMCL signal and is not easy to quantify at commonly used clinical field strengths (18). The present study focuses on muscle fiber-type-related effects on the IMCL content. Since the tCr concentration is also dependent on the muscle fiber type (19), we chose to express both the IMCL and $\mathrm{tCr}$ as a percentage of the water signal from the same voxel.

Both the single-voxel experiments at the different voxel positions and the SI experiment showed that IMCL is inhomogeneously distributed within the TA. The distribution of IMCL over the TA cross section was not random, but emerged in a pattern very similar to the distribution of the type I, IIa, and IIb muscle fibers. This was corroborated by the relations between the different muscle fiber types and the IMCL content (Fig. 6a-c). It has been shown that oxidative muscle fibers can contain up to three times more IMCL than glycolytic type IIb fibers $(10,11)$, and therefore it is most likely that the typical muscle fiber type distribution in the TA accounts for the pronounced IMCL distribution. Thus far, rat muscles have mostly been studied as a whole and classified as predominantly oxidative (e.g., SOL), glycolytic (e.g., TA), or intermediate (e.g., extensor digitorum longus). Our results confirm the muscle fiber type regionalization of the rat TA described by Wang and Kernell (12). Although it has been classified as a "glycolytic" muscle, the rat TA muscle contains a small amount of oxidative type I and fast oxidative type IIa muscle fibers that are clustered close to the tibia bone (12). Although the amount of oxidative muscle fibers is modest relative to the total number of fibers, the pronounced regionalization of oxidative fibers within the TA profoundly affects the IMCL content measured.

Several issues complicate the comparison of these results with previous IMCL studies in Wistar rats. Besides the different methods used to measure and quantify the IMCL content, the positioning of the voxels also differs among the studies. The voxel position within the rat TA in the studies of Neumann-Haefelin et al. $(6,7)$ is not identical to any of the voxel positions used in our study. However, the voxel position used by these researchers is in a region close to the skin that contains hardly any type I and only a few type IIa muscle fibers (personal observation during histological analyses) and therefore can best be compared with our voxel position 1. When the IMCL content reported in our study is normalized to the tCr signal instead of expressed as a percentage of the water signal, then voxel position 1 contains $\sim 30 \%$ of the IMCL signal of the SOL (data not shown), which is in good agreement with the results of Neumann-Haefelin et al. $(6,7)$.

Neumann-Haefelin et al. (6) described various determinants of IMCL concentrations in rat hindleg muscles, including age, strain, gender, and muscle type, and emphasized the need to take these factors into account when studying diseases such as insulin resistance and diabetes. Our results point to the voxel position when applying
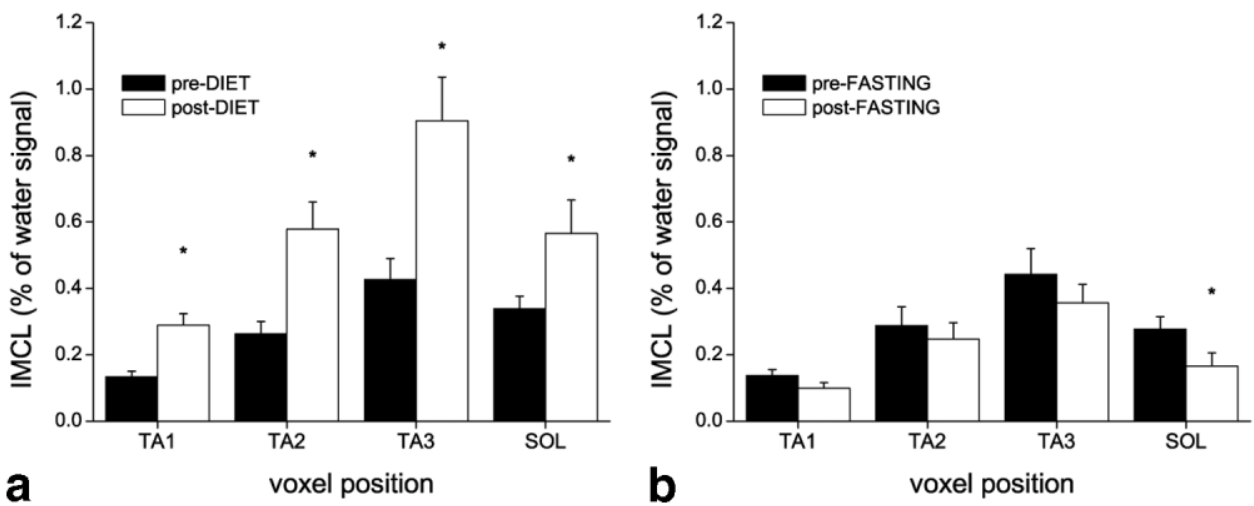

FIG. 7. IMCL content of the different voxel positions in the TA and the SOL (a) before and after 7 days of high-fat diet $(N=6)$, and $(\mathbf{b})$ before and after $15 \mathrm{hr}$ of overnight fasting $(N=5)$. ${ }^{\star} P<0.05$ relative to IMCL content before dietary intervention. 
localized single-voxel ${ }^{1} \mathrm{H}$ MRS as another determinant of the IMCL content in rodent muscle. In practice, the voxel is positioned manually, with the guidance of anatomical landmarks such as the shape of the TA cross section and the tibia bone. This raises the question as to how reproducible the voxel positioning and thus the IMCL measurement actually are. We have shown that the coefficient of variance of the IMCL content is $\sim 16 \%$ for each voxel position in the TA, covering both day-to-day variations in the animal as well as the variation introduced by the positioning of the voxels. The typical pattern of IMCL distribution was constant in the midbelly region of the TA (data not shown), which made the positioning of the voxel in the longitudinal direction far less critical than that in the cross-section of the muscle.

The described inhomogeneous distribution of different muscle fiber types is a key feature of rodent TA muscle. Besides the TA, other rodent muscles are also known to display a pronounced regionalization of their different fiber types. For example, rat gastrocnemius muscle is often analyzed by separating the "white" (glycolytic) part from the "red" (mixed or oxidative) part (20). To our knowledge, ${ }^{1} \mathrm{H}$ MRS has not been used to study IMCL content in rat gastrocnemius muscle. The complex muscle fiber alignment of the gastrocnemius muscle is not ideal for IMCL determination. However, if one were to apply single-voxel ${ }^{1} \mathrm{H}$ MRS to rodent gastrocnemius muscle, one could expect a similar pronounced regionalization of the IMCL content as described in the present study for TA, because of its inhomogeneous fiber type distribution.

We investigated whether a dietary intervention could change the IMCL distribution within the TA, because it has been shown that, e.g., starvation affects IMCL levels in a muscle-specific manner (7). Seven days of high-fat feeding increased the IMCL content in the TA and SOL by more than $100 \%$ and approximately $70 \%$, respectively. However, IMCL was homogeneously increased within the TA and therefore the distribution of IMCL was not changed. Fifteen hours of fasting decreased the IMCL content in the SOL by $40 \%$, but did not cause a significant change in the IMCL levels in the TA. These results appear to contrast with previous reports indicating an increase of IMCL in glycolytic muscles (TA and extensor digitorum longus) and a constant level of IMCL in oxidative muscle (SOL), measured at 12, 24, 48, and $72 \mathrm{hr}$ of starvation (7). Our group size was rather small $(N=5)$, and in contrast to the high-fat diet, the results of the fasting intervention were not consistent for all animals. Some animals showed an increase in IMCL in the TA, whereas others showed a decrease in IMCL following the fasting period. On average this resulted in unchanged IMCL levels in the TA muscle. It is tempting to speculate that the fasting procedure did not induce the expected increase in plasma FFA in all animals; however; we do not have data on the plasma FFA levels.

In conclusion, the variation of the IMCL content within the TA was strongly related to the muscle fiber type distribution and differed by up to a factor of 3 for different voxel positions. Despite changes in the IMCL content due to high-fat feeding and fasting, the typical pattern of IMCL distribution within the rat TA persisted. Therefore, positioning the voxel of interest is a crucial factor when using single-voxel ${ }^{1} \mathrm{H}$ MRS to study IMCL in rodents.

\section{ACKNOWLEDGMENT}

The authors thank Dr. Gustav J. Strijkers for assistance with the processing of the SI data.

\section{REFERENCES}

1. Machann J, Haring H, Schick F, Stumvoll M. Intramyocellular lipids and insulin resistance. Diabet Obes Metab 2004;6:239-248.

2. Yu C, Chen Y, Cline GW, Zhang D, Zong H, Wang Y, Bergeron R, Kim JK, Cushman SW, Cooney GJ, Atcheson B, White MF, Kraegen EW, Shulman GI. Mechanism by which fatty acids inhibit insulin activation of insulin receptor substrate-1 (IRS-1)-associated phosphatidylinositol 3-kinase activity in muscle. J Biol Chem 2002;277:50230-50236.

3. Kuhlmann J, Neumann-Haefelin C, Belz U, Kramer W, Juretschke HP, Herling AW. Correlation between insulin resistance and intramyocellular lipid levels in rats. Magn Reson Med 2005;53:1275-1282.

4. Schick F, Eismann B, Jung WI, Bongers H, Bunse M, Lutz O. Comparison of localized proton NMR signals of skeletal muscle and fat tissue in vivo: two lipid compartments in muscle tissue. Magn Reson Med 1993;29:158-167.

5. Boesch C, Slotboom J, Hoppeler H, Kreis R. In vivo determination of intra-myocellular lipids in human muscle by means of localized $1 \mathrm{H}$ MR-spectroscopy. Magn Reson Med 1997;37:484-493.

6. Neumann-Haefelin C, Kuhlmann J, Belz U, Kalisch J, Quint M, Gerl M, Juretschke HP, Herling AW. Determinants of intramyocellular lipid concentrations in rat hindleg muscle. Magn Reson Med 2003;50:242-248.

7. Neumann-Haefelin C, Beha A, Kuhlmann J, Belz U, Gerl M, Quint M, Biemer-Daub G, Broenstrup M, Stein M, Kleinschmidt E, Schaefer HL, Schmoll D, Kramer W, Juretschke HP, Herling AW. Muscle-type specific intramyocellular and hepatic lipid metabolism during starvation in wistar rats. Diabetes 2004;53:528-534.

8. Vermathen P, Kreis R, Boesch C. Distribution of intramyocellular lipids in human calf muscles as determined by MR spectroscopic imaging. Magn Reson Med 2004;51:253-262.

9. Hwang JH, Pan JW, Heydari S, Hetherington HP, Stein DT. Regional differences in intramyocellular lipids in humans observed by in vivo 1H-MR spectroscopic imaging. J Appl Physiol 2001;90:1267-1274.

10. Malenfant P, Joanisse DR, Theriault R, Goodpaster BH, Kelley DE, Simoneau JA. Fat content in individual muscle fibers of lean and obese subjects. Int J Obes Relat Metab Disord 2001;25:1316-1321.

11. Van Loon LJ, Koopman R, Stegen JH, Wagenmakers AJ, Keizer HA, Saris WH. Intramyocellular lipids form an important substrate source during moderate intensity exercise in endurance-trained males in a fasted state. J Physiol 2003;553:611-625.

12. Wang LC, Kernell D. Proximo-distal organization and fibre type regionalization in rat hindlimb muscles. J Muscle Res Cell Motil 2000;21:587-598.

13. Garwood M, DelaBarre L. The return of the frequency sweep: designing adiabatic pulses for contemporary NMR. J Magn Reson 2001;153:155177.

14. de Graaf RA, Nicolay K. Adiabatic water suppression using frequency selective excitation. Magn Reson Med 1998;40:690-696.

15. Vanhamme L, van den BA, Van Huffel S. Improved method for accurate and efficient quantification of MRS data with use of prior knowledge. J Magn Reson 1997;129:35-43.

16. Kuhlmann J, Neumann-Haefelin C, Belz U, Kalisch J, Juretschke HP, Stein M, Kleinschmidt E, Kramer W, Herling AW. Intramyocellular lipid and insulin resistance: a longitudinal in vivo (1)H-spectroscopic study in Zucker diabetic fatty rats. Diabetes 2003;52:138-144.

17. Korach-Andre M, Gao J, Gounarides JS, Deacon R, Islam A, Laurent D. Relationship between visceral adiposity and intramyocellular lipid content in two rat models of insulin resistance. Am J Physiol Endocrinol Metab 2005;288:E106-E116.

18. Schrauwen-Hinderling VB, Van Loon LJ, Koopman R, Nicolay K, Saris WH, Kooi ME. Intramyocellular lipid content is increased after exercise in non-exercising human skeletal muscle. J Appl Physiol 2003;95: $2328-2332$.

19. Brault JJ, Terjung RL. Creatine uptake and creatine transporter expression among rat skeletal muscle fiber types. Am J Physiol Cell Physiol 2003;284:C1481-C1489.

20. Ferreira LD, Xu D, Palmer TN, Fournier PA. Effect of impaired glucose uptake on postexercise glycogen repletion in skeletal muscles of insulin-treated streptozotocin-diabetic fasted rats. Metabolism 2005;54: 1420-1427. 